Review

\title{
Critical review on microextraction techniques used in determination of histamine in food samples
}

\author{
G. D. T. M. Jayasinghe ${ }^{1} \cdot$ B. K. K. K. Jinadasa ${ }^{1} \cdot$ Pawel Pohl $^{2}$. Aydi Abdelkarim ${ }^{3,4}$
}

Received: 28 October 2021 / Accepted: 27 December 2021

Published online: 03 February 2022

(c) The Author(s) 2022 OPEN

\begin{abstract}
Biogenic amines are basic nitrogen compounds, which are formed due to the decarboxylation of amino acids or/and the amination and the transformation of aldehydes and ketones. Histamine is a heterocyclic biogenic amine present in a wide range of food products, including fish and fishery products, meat and their products, and other dairy products and beverages. The present review summarizes the innovative sample preparation methods for the extraction of histamine from various sample matrices. Both solid phase and liquid phase based microextraction techniques are used for extracting histamine, but the latter ones are the most popular. Currently, all the sample preparation techniques for the histamine extraction fulfil the demand of green chemistry standards. These sample preparation procedures are presented in the present review along with their special features, advantages and disadvantages.
\end{abstract}

\section{Highlights}

1. Classical extraction techniques and detection methods for histamine in food samples.

2. Classification of solid sorbent based and liquid based microextraction techniques.

3. Microextraction techniques based liquid phase and solid sorbent assessments for histamine extraction in food samples.

4. Advantages and disadvantages of microextraction techniques compared with classical extraction techniques.

Keywords Green chemistry · Microextraction · Histamine · Sample preparation

$\triangle$ G. D. T. M. Jayasinghe, thilinimjayasinghe@gmail.com | ${ }^{1}$ Analytical Chemistry Laboratory, National Aquatic Resources Research and Development Agency (NARA), Colombo-15, Sri Lanka. ${ }^{2}$ Department of Analytical Chemistry and Chemical Metallurgy, Faculty of Chemistry, Wroclaw University of Science and Technology, Wybrzeze St. Wyspianskiego 27, 50-370 Wrocław, Poland. ${ }^{3}$ Preparatory Institute for Scientific and Technical Studies, La Marsa (IPEST), Carthage, Tunisia. ${ }^{4}$ French School in Düsseldorf, Graf-Recke-Street 220 , 40237 Düsseldorf, Germany. 


\section{Introduction}

Biogenic amines (BAs) are basic nitrogen compounds, which are formed by the decarboxylation of amino acids or/and amination and the transformation of aldehydes and ketones [1]. According to the chemical structure of BAs, they are classified into aliphatic (putrescine, cadaverine, spermine, spermidine), aromatic (tyramine, phenylethylamine) and heterocyclic (histamine, tryptamine) [2]. The precursors and the structures of main BAs are shown in Table 1. Based on the amine group classification, there are three groups of biogenic amines, i.e., (i) monoamine (phenylethylamine, tyramine, methylamine, ethylamine, isopentylamine, and ethanolamine), (ii) diamine (histamine, tryptamine, serotonin, putrescine, and cadaverine), and (iii) polyamine (spermine, spermidine, and agmatine). Histamine, also known as 2-[4-imidazolyl] ethylamine or 2-[1 H-imidazol-4-yl]ethanamine, is a causative agent of both the histamine intoxication and the histamine intolerance. Based on its chemical structure and number of amine groups, histamine is classified as a heterocyclic diamine [3]. There is another classification of BAs according to the physiological (psychoactive and vasoactive) effects on humans. The vasoactive amines such as tyramine, tryptamine and $\beta$-phenylanine act on the vascular system, and psychoactive amines such as histamine, putrescine, and cadaverine act on the nervous system [4].

BAs are present in a wide range of food products, including fish and fishery products, meat and their products, dairy products, beer, wine, nuts, fruits, vegetables, and chocolate [5]. Among different BAs histamine is the most important one. It is synthesized from histidine by pyridoxal phosphate (vitamin B-6) containing L-histidine decarboxylase. The scombroid poisoning, also called the histamine fish poisoning, continues a major problem for the seafood safety. It is characterized by a variety of symptoms similar to allergic reactions, but the exact role of histamine for the scombroid poisoning is not known [6]. The histamine poisoning can be easily distinguished from the food allergy based on the following points: (1) the lack of a previous history of allergic reactions to the incriminated food, (2) the high attack rate in the group outbreaks, and (3) the measure of high levels of histamine in the incriminated food [7]. The oral administration of pure histamine does not cause any systemic effects as compared to the scombroid. This is because a pure dose of histamine is inactivated in the intestine before entering the portal circulation, converted from the enteric flora. There are some additional combined effects of other BAs and substances related with histamine that affect the scombroid poisoning. It is discovered that the histamine content and the scombroid poisoning are correlated, but there is no clear dose response relationship between the oral administration of histamine and a histamine dose consumed with digested fish and fishery products [8-10]. Countries like Japan, the United State of America, Great Britain report that the incident of the histamine poisoning has been ongoing since 1970. Less frequent outbreaks are reported in some other countries, including New Zealand, Norway, Germany, France, Sri Lanka, Canada, Sweden, Czechoslovakia, Indonesia, Australia, Netherland, Egypt, and South Africa [11, 12]. The largest histamine outbreak yet has been recorded in Japan (in 1973); it involved 2656 cases due to the consumption of dried horse mackerels [12]. The cases of the histamine poisoning recorded every year throughout the world are very important to understand the health effects coming from this amine toxin [13-15].

The symptoms associated with the histamine poisoning are similar to other allergic conditions in the human body and usually mild or some short-duration symptoms are observed when an excessive amount of histamine is taken from the seafood. These symptoms typically occur within 20-30 min after the histamine contaminated food consumption and most commonly include the following symptoms: facial flushing, abdominal pain, headaches, diarrhea, itching, red rashes, hypertension, and palpitation $[9,16]$. The risk of the histamine poisoning can be controlled by applying the basic good manufacturing and the hygienic practices associated with the appropriate Hazard Analysis Critical Control Point guidelines [17]. European Commission (EC), United States Food and Drug Administration (FDA), and other corresponding institutions in such countries like Australia [18], South Africa, Canada [19], Switzerland, Finland [20], or Brazil [21] have implemented the regulation limits for histamine in fish and fishery products.

Table 2 shows the regulation limits for histamine in fish given in the EC [22] and FDA [23]. As an example, a minimum of 9 samples should be analysed based on the EU/EC regulation and the average value of histamine in these samples should be less than $100 \mathrm{mg} / \mathrm{kg}$ while in 2 of these samples it can exceed $100 \mathrm{mg} / \mathrm{kg}$ but should not be higher than $200 \mathrm{mg} / \mathrm{kg}$.

Currently, chromatography and spectroscopy techniques are routinely used for the histamine determination. In particular, the High-Performance Liquid Chromatography (HPLC) separation combined with different kinds of detectors play the most important role in the histamine quantification. These detection methods are Molecular Fluorescence Spectroscopy [24, 25], Ultraviolet Absorption Spectroscopy [26, 27], diode array UV-Vis, Mass Spectrometry (MS or MS-MS) [28, 29], Thin Layer Chromatography (TLC) [30, 31], colorimetry [32], fluorimetry [33], lon Mobility Spectrometry (IMS) [34], Filter Paper Electrophoresis [35], commercially available histamine test kits based on enzymatic methods [36, 37], Gas Chromatography-Flame lonization and Detector (GC-FID) Gas Chromatography-Mass Spectrometry (GS-MS) [38]. 
The sample preparation is a basic and crucial step that enables to enrich the analytes and remove the interferences. Food samples are very complex and often contain proteins, fats, salts, acids and bases, and food additives, etc. For that reason, novel sample preparation techniques, highly selective and with the enhanced extraction efficiency, are needed to be applied to pre-treat the analysed food matrices [39]. Nevertheless, the pre-concentration is often necessary due to low concentrations of the analyte in the samples and an insufficient sensitivity that is provided by given detection systems. Many approaches to the pre-concentration/extraction of histamine from fish, wine, and cheese samples have been proposed. The classical extraction and other techniques such as Solid-Phase Extraction (SPE) [40], Liquid-Liquid Extraction (LLE) [41], Ultra-sound-Assisted Extraction (USA) and Microwave-Assisted Extraction (MAE) [42] are widely used in this case. But in recent years, microextraction techniques have become more popular because they offer new possibilities, including a higher reduction of tSe extraction time, as well as a considerably lower sample weight/volume and a lower extractant/solvent volume.

To eliminate the drawbacks of the classical extraction methods and their impacts on the environment, new methods have been developed. The microextraction based on solid sorbents and the microextraction based on liquid extractants combine both groups of methods, developing miniaturized green chemistry extraction approaches to the separation, the pre-concentration, and the reliable determination of histamine, moving at the same time all these novel techniques toward the simplification and the automation [43].

The current review is focused on the microextraction techniques, which are used for the histamine extraction from food products. Some of the new methodologies, consisting of solid sorbent-based and liquid-based microextraction techniques, are surveyed as miniaturized sample preparation techniques for the histamine separation and pre-concentration. The discussion included in the present review is mainly focused on the availability of different microextraction techniques for the target identification, pre-concentration, and/or clean-up purpose, and the selected analytical figures of merit (enrichment factors, Limits of Detection (LOD) and Limits of Quantification (LOQ), analytical recoveries) that they offer.

\section{Microextraction techniques based on solid sorbents}

Solid sorbent-based (solid phase) microextraction techniques, so-called Solid-Phase Microextraction (SPME), are carried out without any solvent necessary for the isolation step of the analytes, but it also requires a little amount of solvents or no solvents at all for the desorption step [44]. According to the extraction procedure, this technique can be categorized into three major fields: Fibre-based solid-phase microextraction, Stir Bar Sorptive Extraction (SBSE) and Microextraction in a Packed Syringe (MEPS) [42] (Fig. 1). It must be noted that all these techniques may not be used for the extraction of histamine from food samples. SPME is a fast, universal, and solvent-less separation/pre-concentration technique that is used for the sample preparation and analysis, mainly prior to the Gas Chromatography (GC) or HPLC separation and detection methods. The effectiveness of the analyte pre-concentration using SPME depends on many parameters such as the type of a fiber, the sample stirring method, the extraction time, and the sample ionic strength [45].

The pre-concentration efficiency depends on the type of a fibre and its thickness (amount of the fibre). The general rule is that "polar compounds are sorbed on polar fibres while nonpolar compounds on nonpolar fibres" [46]. SBSE is another type of the solid sorbent-based microextraction technique introduced in 1999. This technique has been developed to extract the organic analytes from liquid samples and it is based on the sorption of the analytes onto a thick film such as polydimethylsiloxane (PDMS) coated on an iron stir bar [47]. The SBSE technique is applied to obtain low LODs of the analytes when combining it with the thermal desorption GC-MS detection method [48]. As an alternative, the analyte extract obtained with the aid of SBSE can be injected into a Liquid Chromatography (LC) system [47]. MEPS is a new miniaturized solid sorbent-based microextraction technique that can be connected online to the GC or LC systems without any special modification [49]. MEPS can be handled with a small sample volume (10 $\mu \mathrm{L})$ as well as a large volume $(1000 \mu \mathrm{L})$ and, as compared to LLE and SPE techniques, it reduces the sample preparation time, and the organic solvent volume used, having higher extraction efficiencies and recoveries of the target analytes [50].

The SPME technique for the determination of histamine has been applied using different types of commercially available fibres such as PDMS and polyvinylpyrrolidone, etc. [51, 52]. In addition, the SPME technique, working both in direct immersion [51] or headspace (HS) [34,53] modes, is successfully applied for the GC-MS and ion mobility spectroscopy detection. 
Table 1 Precursors and structures of the main biogenic amines

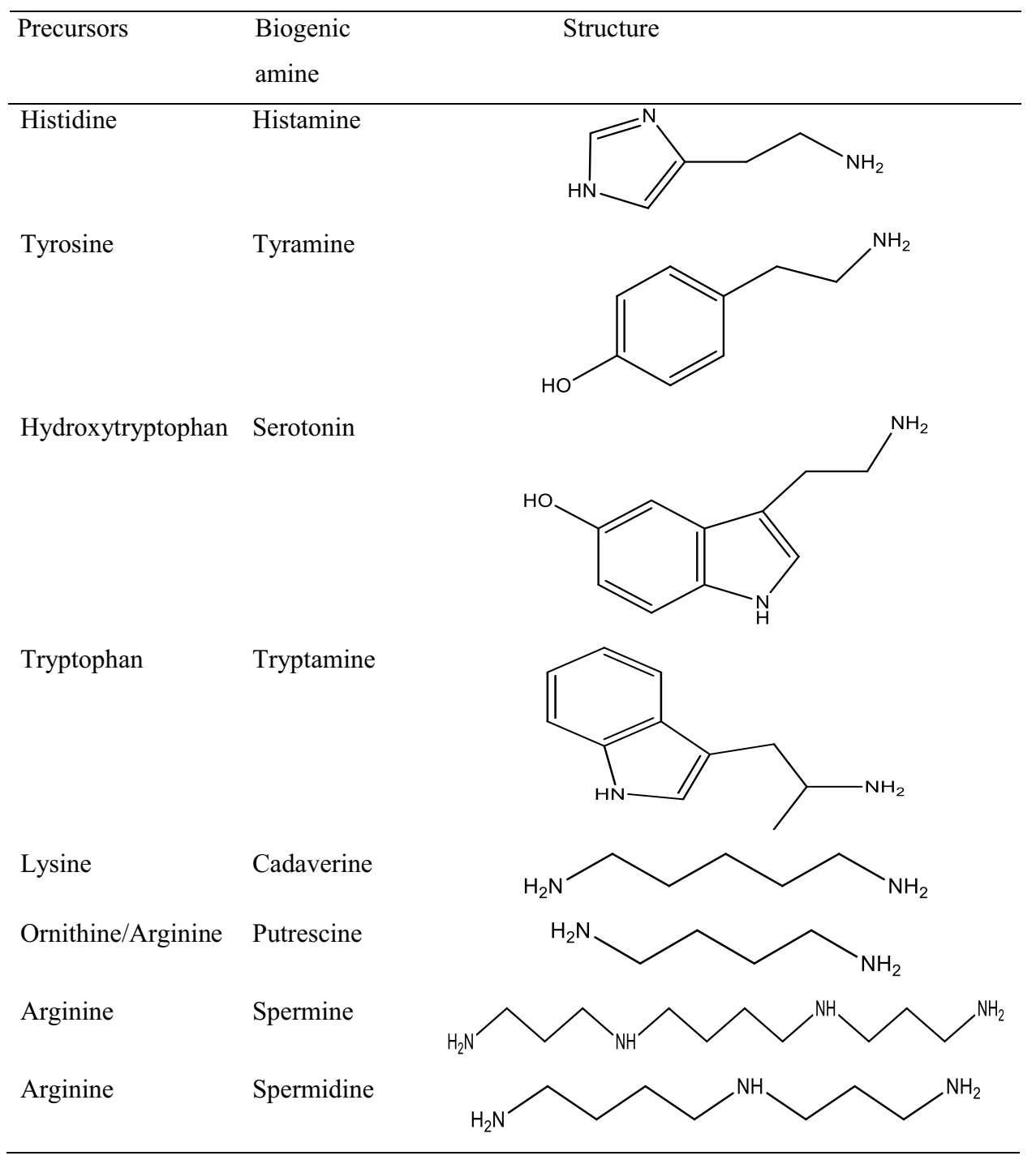

Table 3 shows the recently used solid sorbent-based microextraction techniques for the determination of histamine in different food matrices.

In a generic term, Micro Solid Phase Extraction ( $\mu$-SPE) has been used when a reduced amount of sorbents, and/or microsized or nanosized materials were applied for the extraction [54]. There are several $\mu$-SPE techniques reported in the literature, including Dispersive Micro Solid Phase Extraction (D- $\mu-S P E)$, membrane protected micro solid phase extraction (MP- $\mu$-SPE), pipette tip micro solid phase extraction (PT- $\mu$-SPE) and spin column micro solid phase extraction (SC- $\mu$-SPE). However, $\mu$-SPE methods are suitable only for the analyte extraction from the liquid sample, while the SPME method can be applied for the gas samples too [55]. Basheer et al. [56] studied eight different hydrozone based ligands for the $\mu$-SPE determination of 6 BAs, i.e., tryptamine, phenylethylamine, putrescine, histamine, tyramine and spermidine, in orange juice. Benzophenone 2,4-dinitrophenylhydrazone was selected as best sorbent. Moreover, they observed that $\mu$-SPE demonstrated rather low recoveries of the analytes (81.4-86.3\%) as compared to those obtained with SPE (90.3-92.4\%), but showed a quite high enrichment factor for them (314).

Most of the researchers use the HPLC and GC-MS techniques for the determination of histamine. A very interesting procedure has been proposed by Alizadeh et al. [53], who used IMS, a novel analytical technology, for the separation and the identification of ionized molecules. The theory behind the IMS is that the ionized molecules drift in the gas phase based on their mobility in the electric field at the atmospheric pressure. The required time, the travel length and drift of the ions depend on their mass, charge distribution and shape [57]. To check the reliability of the HS-SPME-IMS method, samples are analysed with the GC-MS method. As compared to the GC-MS method, the advantages of the HS-SPME-IMS 
Table 2 Regulation limits for the histamine content in fish and fishery products

\begin{tabular}{lrrrr}
\hline Sampling plan & $\mathrm{n}$ & $\mathrm{c}$ & \multicolumn{2}{c}{ Histamine content $(\mathrm{mg} / \mathrm{kg})$} \\
\cline { 4 - 5 } & & & $\mathrm{m}$ & $\mathrm{M}$ \\
\hline European Union & 9 & 2 & 100 & 200 \\
FDA & 18 & 1 & 50 & 500 \\
\hline
\end{tabular}

$\mathrm{n}$, number of comprising the sample unit; $c$, number of sample unit; $m$, the mean value is less or equal; $M$, no value exceeds

method are as follows: very fast analyses (need a millisecond time scale), the high sensitivity and simplicity, the solventfree separation/pre-concentration, the clean separation and good spectra without the presence of matrix interferences, and no need for the derivatization step [53].

Basheer et al. [56] developed another SPME method based on crown ethers and hydrazone used as sorbent materials. In this study, synthesized hydrozone ligands were physically trapped into a sol-gel network and used as the SPME material. The proposed approach resulted in a good enrichment factor for all BAs standards. However, the main disadvantage of this method was low recoveries and the low sensitivity when the method was applied for real samples. The hydrozone ligand was established to have a high extraction efficiency for aliphatic BAs except for both aliphatic and aromatic BAs. Nevertheless, the major advantage of this method was the possibility to reduce the solvent consumption and time; in addition, the new SPME materials were easily fabricated, inexpensive, and reusable, and provided high preconcentration factors [56].

Solid sorbent-based microextraction techniques, specially SPME, have commonly several important advantages as compared to traditional sample preparation techniques; they are rapid, simple, and easy, solventless or completely free of solvent, and selective or specific to extract the analytes. Moreover, SPME can simultaneously be used for the sample clean-up and the extraction of the analytes from the complex matrices [4]. The adsorption and desorption properties of the SPME technique enable to use relatively small amounts of samples for the analysis. The technique can be performed in the field, giving consistent and reliable results even for very low concentrations of analytes in the analysed samples. However, there are some drawbacks to this technique. The fibres used can be operated at relatively low temperatures in GC (generally $240-280^{\circ} \mathrm{C}$ ). They can be broken, while during their operation, coating can be stripped off, and/or swelled in the organic solvent, while the needle can be bent $[58,59]$.

\section{Microextraction techniques based on liquid phase}

In the Liquid Phase Microextraction (LPME) technique, the extraction usually takes place in a microliter amount of a water-immiscible solvent (the acceptor phase). The LPME technique can be divided into three main groups: SDME, Hollow Fibre Liquid-Phase Microextraction (HF-LPME), and Dispersive Liquid-Liquid Microextraction (DLLME). Figure 2 shows the microextraction techniques based on the liquid phase. The common feature of all three LPMEs is that at least one liquid solvent, a most probably microliter scale volume (1-100 $\mu \mathrm{L})$ organic solvent, is used to extract the analytes of interest. The main drawback in LPME is the need for the stabilization of the liquid extracting phase. To overcome this issue, some holders were introduced to house this phase and increase the contact surface [60].

Classical sample extraction methods such as LLE and SPE have either serious drawbacks, including long extraction times and a high consumption of toxic solvents, etc. To overcome these problems, Liu and Dasgupta [61] were the first who report a novel "drop-in drop" system, where a micro drop of a water-immiscible organic solvent is suspended in a large aqueous drop [61]. At the same time Jeannot and Cantwell [62] introduced a new solvent microextraction technique, which used micro drops of organic solvent suspended at the end of a Teflon rod immersed in a stirred aqueous sample solution [62]. He and Lee [63] also introduced a novel technology of the LPME using a microsyringe and a GC sample injector and it is now a very attractive alternative for SPME, offering such facilities and advantages such as simplicity, low costs, and high enrichment efficiencies.

In the case of the Single Drop Microextraction (SDME) technique, which is based on the usage of a single drop of the acceptor phase, the acceptor phase may be immersed directly into a sample solution. It is called the Direct-Immersion Single Drop Microextraction (DI-SDME) technique. Sometimes, the acceptor phase is suspended over the sample in the headspace, and this is known as the Head-Space Single-Drop Microextraction (HS-SDME) technique. The SDME technique is older than the LPME technique and can be broadly classified into two-phase and three-phase techniques. The SDME 


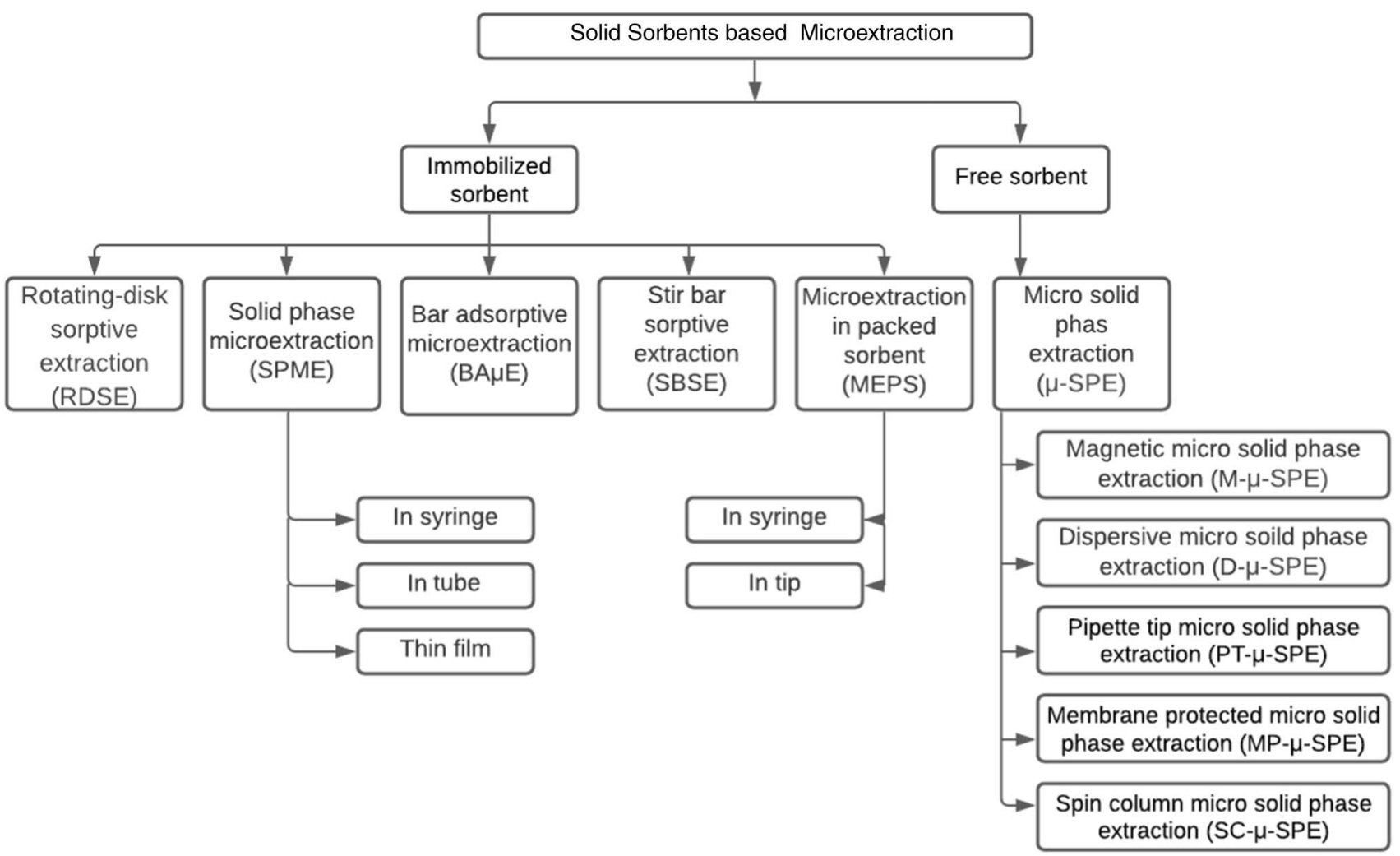

Fig. 1 Microextraction techniques based on solid sorbents [55, 80-82]

technique is simple, cost-effective, and has a lot of useful applications [64]. However, SDME technique needs different apparatus for the extraction of the target analytes; and the need for the sample injection is another disadvantage. In addition, when higher stirring speeds are used, it is difficult to form stable droplets $[58,65]$.

The HF-LPME technique is based on the use of disposable propylene porous hollow fibres filled with small amounts of selected extracting solutions (the acceptor phase) immersed in an aqueous solution (the donor phase). This technique has several benefits, i.e., cost-effectiveness, efficient cleaning, analysis speed, and high enrichment factors. The main drawbacks of the HF-LPME technique are the ability to absorb hydrophobic substances or the formation of air bubbles on the surface of the fibres during the extraction, which can affect the reproducibility of the results [66]. DLLME is another type of the liquid-based microextraction technique, which offers a fast injection of a suitable mixture of solvents, one being the extraction solvent, and the other being the dispersive solvent for the analytes. After adding the dispersive solvent, a cloudy solution containing the fine droplets of the extraction solvent (fully dispersed in the aqueous phase) is formed. Finally, the extraction phase can be accumulated at the bottom of an extraction container by the centrifugation [59].

As modern analytical chemistry aims at reducing the analysis time, the sample preparation procedures became a bottleneck of the analysis. However, the extraction of histamine from different food matrixes can be easily achieved by the liquid phase-based microextraction techniques. Table 4 shows the liquid phase-based microextraction techniques which were later used for the histamine extraction. The most popular liquid phase microextraction technique was DLLME, which was applied individually or in combination with other techniques. The combined extraction techniques such as Vortex Assisted Extraction (VA), Ultra-Sound-Assisted (USA) extraction, Electro Membrane Extraction (EME), and MicrowaveAssisted (MA) Extraction were combined with DLLME to extract histamine to increase the pre-concentration factor and the recovery percentage for this analyte.

The USA-DLLME technique was successfully introduced for the histamine extraction from different sample matrices i.e., beer [67], fermented fish [68], and foodstuffs (cheese, yogurt, rice wine, beer, and ham) [69]. Different extraction solvents (methanol, acetonitrile, and acetone) and different dispersive solvents (toluene, 1-octanol and chloroform) were used but the quantitative (88-110\%) recoveries were obtained in this case. Mohammadi et al. [70] presented MADLLME for the histamine extraction from cheese. They highlighted that the microwave energy was absorbed by polar 
Table 3 Solid sorbent based microextraction techniques applied for the extract of histamine

\begin{tabular}{|c|c|c|c|c|c|c|c|}
\hline Sample matrix & $\begin{array}{l}\text { Microextraction } \\
\text { technique }\end{array}$ & Sorbent & $\begin{array}{l}\text { Instrumental } \\
\text { technique }\end{array}$ & LOD & $\mathrm{EF}$ & AR\% & Refs. \\
\hline Canned fish & HS-SPME & Ppy-DBS & GC-MS & $0.6-1 \mathrm{ng} / \mathrm{mL}$ & & $91-97$ & {$[34]$} \\
\hline Orange juice & SPME & Hydrazone & HPLC-UV & $8.22 \mathrm{ng} / \mathrm{L}$ & $94-460$ & 92.4 & {$[56]$} \\
\hline Wine & SPME & Fiber material & GC-MS & $0.521 \mu \mathrm{g} / \mathrm{L}$ & - & $82-97$ & {$[83]$} \\
\hline Beer & SPME & PVP & HPLC & $0.17 \mu \mathrm{g} / \mathrm{mL}$ & - & $90.2-92.2$ & {$[52]$} \\
\hline $\begin{array}{l}\text { Fish sauce, Soy sauce, } \\
\text { Ketchup, Orange juice }\end{array}$ & SPME & 4-HPMB & HPLC-UV & $0.003-0.031 \mu \mathrm{g} / \mathrm{L}$ & - & $8.5-73.3$ & {$[84]$} \\
\hline Canned fish & HS-SPME & Ppy -fiber & IMS & $3 \mathrm{ng} / \mathrm{g}$ & - & $94-96$ & {$[53]$} \\
\hline Fish & DI-SPME & PDMS/DVB & GC-MS & $2.98-45.3 \mu \mathrm{g} / \mathrm{L}$ & & $78.9-110$ & {$[51]$} \\
\hline Orange juice & $\mu$-SPE & $\mathrm{BPDH}$ & HPLC-UV & $8.22 \mathrm{ng} / \mathrm{L}$ & 314 & 86.3 & {$[56]$} \\
\hline Smakked fish & SPME & $\mathrm{Fe}_{3} \mathrm{O}_{4}-\mathrm{g}-\mathrm{GO}$ & HPLC-UV & $0.017 \mathrm{mg} / \mathrm{L}$ & _ & $98-101$ & {$[85]$} \\
\hline
\end{tabular}

$E F$, extraction efficiency; AR, analytical recovery; LOD, limit of detection, HS-SPME, head space solid phase microextraction; PPy-DBS, polypyrrole-dodecyl-benzenelsulfonate; GC-MS, gas chromatography mass spectrometry; HPLC-UV, high performance liquid chromatography ultraviolet detection; TFME, thin film microextraction; FL, fluorescence detection; PVP, polyvinylpyrrolidone; IMS, ion mobility spectrometry; Ppy, polypyrrole fiber; DI, direct immersion; PDMS/DVB, polydimethylsiloxane/divinylbenzene; BPDH-benzophenone 2,4-dinitrophenylhydrazone, 4-HPMB- 4-hydroxy-Na-[(E)-(2-hydroxyphenyl)methylidene]benzohydrazide; GO, Graphne oxide

compounds, hence the temperature and the pressure of the process increased, causing the target molecules (histamine) to be rapidly transferred from the sample matrix to the liquid phase. The mentioned MA-DLLME technique has several advantages, considering the increased recovery, low volumes of hazardous organic solvents used, and a simple and low time-consuming course of the process. This technique provided the quantitative recovery of histamine and a very high enrichment factor of this analyte [70].

Kamankesh et al. [71] applied a novel sample preparation procedurebased on EME and $\mu$-dispersive liquid-liquid microextraction to simultaneously determine tyramine, histamine, putrescine, and cadaverine in canned fish [71]. The EME technique was introduced in 2006 and enabled to extract the target analytes from aqueous sample solutions and transfer them through a Supported Liquid Membrane (SLM) just to enter into aqueous or organic acceptor solutions $[72,73]$. The proposed extraction technique offered high sensitivity, good selectivity, and the quantitative recovery of histamine, it is a very high enrichment factor. In addition, it could be easily and fastly used for the simultaneous determination of four BAs, including histamine [71].

Ionic Liquids (ILs) are more environmentally friendly green solvents due to their low toxicity and low volatility as compared to organic solvents. They show dual micellar transitions in aqueous solutions, acting as both dispersers and sensitivity enhancers. ILs represent a novel solvent class that is widely used in the field of chemistry due to their good thermal and chemical stability, good extractability and high separation power of many compounds, low flammability, high viscosity and high electrical conductivity [74-76]. Moreover, due to their micellar properties, they are used as the disperser solvents in DLLME and better dispers the organic solvents in the aqueous phase than the commonly used solvents [77]. The main disadvantage of ILs is their high cost [78]. The ILs based DLLME technique was proposed for the histamine determination by Cao et al. [79] and Elik et al. [77]. They successfully applied this microextraction technique for the recovery and pre-concentration of histamine from wine, fish, and meat, and obtained analytical recoveries of histamine $>90 \%$ and very low LODs of this analyte.

Fig. 2 The microextraction techniques based on the liquid phase [80]

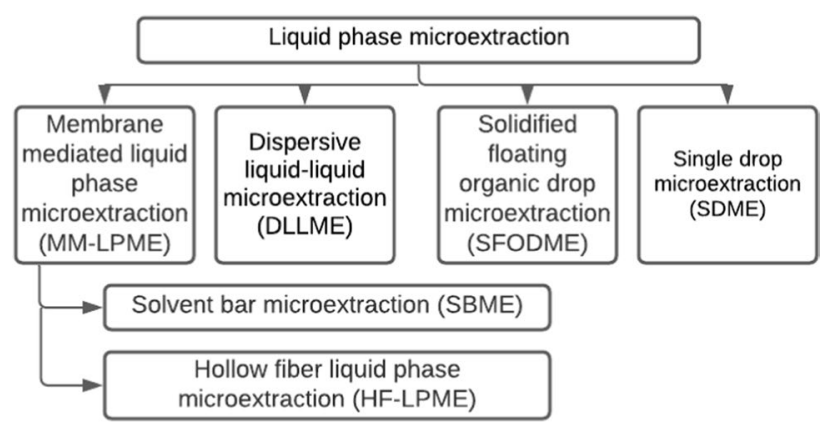




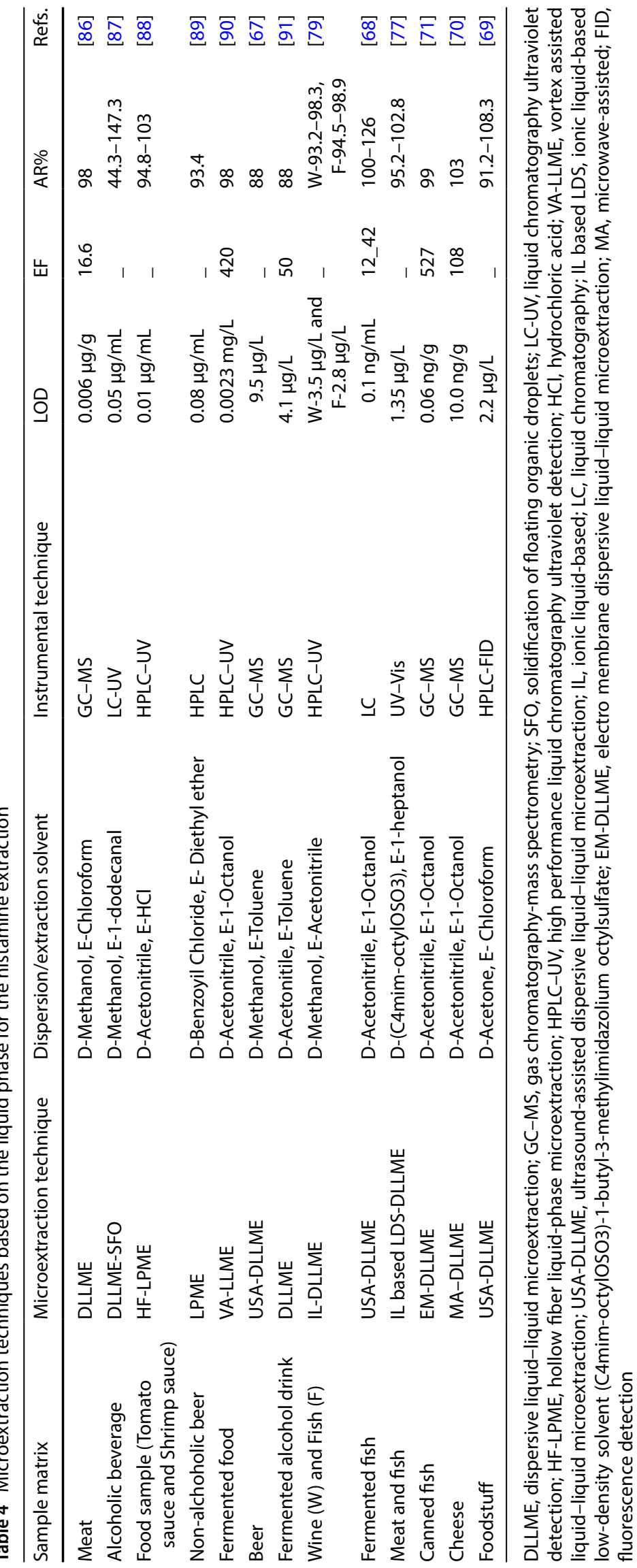


Many analytical procedures based on microextraction techniques have been developed for the sample preparation and the determination of histamine in various sample matrixes. When considering all types of the microextraction techniques applied for histamine, it must be taken into account that all applications follow the principles and rules of the green (analytical) chemistry concept. Completely new sorptive materials were synthesized to adsorb histamine and other BAs in addition to the application of ILs as new solvents. This enabled to develop and validate the completely new extraction methods following the green chemistry rules. As evidenced in the present review, the microextraction techniques based on the liquid phase are more popular and diversified for the histamine determination than the solid sorbent-based microextraction techniques. The application of the combined techniques with the liquid phase microextraction techniques certainly gives a real possibility of the much higher enrichment factors and the compatibility with the analytical procedures used for the detection/determination of histamine.

\section{Conclusions}

The term of the microextraction encompasses a group of extraction techniques in which the extraction phase may be solid or liquid. Substantially, the development of these extraction techniques is dictated by the need for smaller $(<100$ $\mu \mathrm{L}$ or $<10 \mathrm{mg}$ ) sample volumes (required and/or available for analysis) as compared to classical extraction methods. The SPME technique has been in a forefront of microextraction research, nevertheless, other microextraction techniques, either with solid or liquid extraction phases, have been also developed in parallel. According to the literature, both microextraction techniques based on the liquid and solid sorbents, used for the histamine extraction and its determination in addition to the LPME technology are more and more popular due to an effective and interference-free utilization of histamine from various sample solutions. Both microextraction techniques are classified as the "green chemistry" sample preparation procedures, with several advantages, such as the reduced extraction time as compared to the other traditional extraction procedures, much lower investment and operational costs, and very good analytical figures of merit, including enrichment factors, high recoveries, lower LODs, and higher sample throughput.

As a conclusion for the future development, it can be honestly stated that the potential of the microextraction is much greater and that the next generation of the analytical scientists will be able to fully take the advantage of its advances, particularly in the case of histamine and other BAs.

Authors' contributions Jayasinghe GDTM: writing and original draft preparation JBKKK: writing, reviewing, \& drawing. PP: reviewing and editing. AA: structuring and supporting. All authors read and approved the final manuscript.

\section{Declarations}

Competing interests The authors declare no competing interests.

Open Access This article is licensed under a Creative Commons Attribution 4.0 International License, which permits use, sharing, adaptation, distribution and reproduction in any medium or format, as long as you give appropriate credit to the original author(s) and the source, provide a link to the Creative Commons licence, and indicate if changes were made. The images or other third party material in this article are included in the article's Creative Commons licence, unless indicated otherwise in a credit line to the material. If material is not included in the article's Creative Commons licence and your intended use is not permitted by statutory regulation or exceeds the permitted use, you will need to obtain permission directly from the copyright holder. To view a copy of this licence, visit http://creativecommons.org/licenses/by/4.0/.

\section{References}

1. Santos MS. Biogenic amines: their importance in foods. Int J Food Microbiol. 1996;29(2-3):213-31.

2. Bardócz S, Grant G, Brown DS, Ralph A, Pusztai A. Polyamines in food-implications for growth and health. J Nutr Biochem. 1993;4(2):66-71.

3. Erdag D, Merhan O, Yildiz B. Biochemical and pharmacological properties of biogenic amines. Biog Amines. 2018;8:1-14.

4. Mohammed Gl, Bashammakh AS, Alsibaai AA, Alwael H, El-Shahawi MS. A critical overview on the chemistry, clean-up and recent advances in analysis of biogenic amines in foodstuffs. TrAC Trends Anal Chem. 2016;78:84-94. https://doi.org/10.1016/j.trac.2016.02.007.

5. Brink B, Damink C, Joosten H, In't Veld JH. Occurrence and formation of biologically active amines in foods. Int J Food Microbiol. 1990;11(1):73-84.

6. Visciano P, Schirone M, Tofalo R, Suzzi G. Histamine poisoning and control measures in fish and fishery products. Front Microbiol. 2014. https://doi.org/10.3389/fmicb.2014.00500. 
7. DeBeer J, Bell JW, Nolte F, Arcieri J, Correa G. Histamine limits by country: a survey and review. J Food Prot. 2021;84(9):1610-28. https:// doi.org/10.4315/jfp-21-129.

8. Hungerford JM. Scombroid poisoning: a review. Toxicon. 2010;56(2):231-43. https://doi.org/10.1016/j.toxicon.2010.02.006.

9. Lehane L, Olley J. Histamine fish poisoning revisited. Int J Food Microbiol. 2000;58(1-2):1-37.

10. Tortorella V, Masciari P, Pezzi M, Mola A, Tiburzi SP, Zinzi MC, et al. Histamine poisoning from ingestion of fish or scombroid syndrome. Case Rep Emerg Med. 2014;2014:482531. https://doi.org/10.1155/2014/482531.

11. Lehane L. Histamine (Scombroid) Fish Poisoning: a review in a risk-assessment framework. National Office of Animal and Plant Health. 1999.

12. Tao Z, Sato M, Zhang H, Yamaguchi T, Nakano T. A survey of histamine content in seafood sold in markets of nine countries. Food Control. 2011;22(3-4):430-2.

13. Kang CR, Kim YY, Lee Jl, Joo HD, Jung SW, Cho SI. An outbreak of scombroid fish poisoning associated with consumption of yellowtail fish in Seoul, Korea. J Korean Med Sci. 2018. https://doi.org/10.3346/jkms.2018.33.e235.

14. Colombo FM, Cattaneo P, Confalonieri E, Bernardi C. Histamine food poisonings: a systematic review and meta-analysis. Crit Rev Food Sci Nutr. 2018;58(7):1131-51. https://doi.org/10.1080/10408398.2016.1242476.

15. Toda M, Yamamoto M, Uneyama C, Morikawa K. Histamine food poisonings in Japan and other countries. Bull Natl Instit Health Sci. 2009;127:31-8.

16. Becker K, Southwick K, Reardon J, Berg R, MacCormack JN. Histamine poisoning associated with eating tuna burgers. JAMA. 2001;285(10):1327-30.

17. Visciano P, Schirone M, Tofalo R, Suzzi G. Histamine poisoning and control measures in fish and fishery products. Front Microbiol. 2014;5:500.

18. Rauscher-Gabernig E, Grossgut R, Bauer F, Paulsen P. Assessment of alimentary histamine exposure of consumers in Austria and development of tolerable levels in typical foods. Food Control. 2009;20(4):423-9.

19. Rai KP, Pradhan HR, Sharma BK, Rijal SK. Histamine in foods: its safety and human health implications. J Food Sci Technol Nepal. 2013;8:1-11.

20. Hu Y, Xia W, Liu X. Changes in biogenic amines in fermented silver carp sausages inoculated with mixed starter cultures. Food Chem. 2007;104(1):188-95.

21. Biji K, Ravishankar C, Venkateswarlu R, Mohan C, Gopal TS. Biogenic amines in seafood: a review. J Food Sci Technol. 2016;53(5):2210-8.

22. Commission E. Microbiological criteria for foodstuffs. Commission Regulation EC No 2073/2005: OJ.L; 2005. p. 1-26.

23. Shakila RJ, Vijayalakshmi K, Jeyasekaran G. Changes in histamine and volatile amines in six commercially important species of fish of the Thoothukkudi coast of Tamil Nadu, India stored at ambient temperature. Food Chem. 2003;82(3):347-52. https://doi.org/10.1016/ S0308-8146(02)00552-6.

24. Ishimaru M, Muto Y, Nakayama A, Hatate H, Tanaka R. Determination of biogenic amines in fish meat and fermented foods using columnswitching high-performance liquid chromatography with fluorescence detection. Food Anal Methods. 2019;12(1):166-75. https://doi. org/10.1007/s12161-018-1349-0.

25. Duflos G, Inglebert G, Himber C, Degremont S, Lombard B, Brisabois A. Validation of standard method EN ISO 19343 for the detection and quantification of histamine in fish and fishery products using high-performance liquid chromatography. Int J Food Microbiol. 2019;288:97-101. https://doi.org/10.1016/j.ijfoodmicro.2018.07.023.

26. Nadeem M, Naveed T, Rehman F, Xu Z. Determination of histamine in fish without derivatization by indirect reverse phase-HPLC method. Microchem J. 2019;144:209-14. https://doi.org/10.1016/j.microc.2018.09.010.

27. Learey JJ, Crawford-Clark S, Bowen BJ, Barrow CJ, Adcock JL. Detection of biogenic amines in pet food ingredients by RP-HPLC with automated dansyl chloride derivatization. J Sep Sci. 2018;41(24):4430-6. https://doi.org/10.1002/jssc.201800455.

28. Czajkowska-Mysłek A, Leszczyńska J. Liquid chromatography-single-quadrupole mass spectrometry as a responsive tool for determination of biogenic amines in ready-to-eat baby foods. Chromatographia. 2018;81(6):901-10. https://doi.org/10.1007/s10337-018-3527-z.

29. Kaufmann A, Maden K. Easy and fast method for the determination of biogenic amines in fish and fish products with liquid chromatography coupled to orbitrap tandem mass spectrometry. J AOAC Int. 2018;101(2):336-41. https://doi.org/10.5740/jaoacint.17-0407.

30. Shalaby AR. Significance of biogenic amines to food safety and human health. Food Res Int. 1996;29(7):675-90.

31. Shakila RJ, Vasundhara T, Kumudavally K. A comparison of the TLC-densitometry and HPLC method for the determination of biogenic amines in fish and fishery products. Food Chem. 2001;75(2):255-9.

32. Patange S, Mukundan M, Kumar KA. A simple and rapid method for colorimetric determination of histamine in fish flesh. Food Control. 2005;16(5):465-72.

33. Adamou R, Coly A, Douabalé SE, Saleck MLOCO, Gaye-Seye MD, Tine A. Fluorimetric determination of histamine in fish using micellar media and fluorescamine as labelling reagent. J Fluoresc. 2005;15(5):679.

34. Parchami R, Kamalabadi M, Alizadeh N. Determination of biogenic amines in canned fish samples using head-space solid phase microextraction based on nanostructured polypyrrole fiber coupled to modified ionization region ion mobility spectrometry. J Chromatogr A. 2017;1481:37-43. https://doi.org/10.1016/j.chroma.2016.12.046.

35. Tao Z, Sato M, Yamaguchi T, Nakano T. Formation and diffusion mechanism of histamine in the muscle of tuna fish. Food Control. 2009;20(10):923-6.

36. Aygün O, Schneider E, Scheuer R, Usleber E, Gareis M, Märtlbauer E. Comparison of ELISA and HPLC for the determination of histamine in cheese. J Agric Food Chem. 1999;47(5):1961-4.

37. Köse S, Kaklıkkaya N, Koral S, Tufan B, Buruk KC, Aydın F. Commercial test kits and the determination of histamine in traditional (ethnic) fish products-evaluation against an EU accepted HPLC method. Food Chem. 2011;125(4):1490-7. https://doi.org/10.1016/j.foodchem. 2010.10.069.

38. Hwang B-S, Wang J-T, Choong Y-M. A rapid gas chromatographic method for the determination of histamine in fish and fish products. Food Chem. 2003;82(2):329-34.

39. Xu C-H, Chen G-S, Xiong Z-H, Fan Y-X, Wang X-C, Liu Y. Applications of solid-phase microextraction in food analysis. TrAC, Trends Anal Chem. 2016;80:12-29. https://doi.org/10.1016/j.trac.2016.02.022. 
40. Brillantes S, Samosorn W. Determination of histamine in fish sauce from Thailand using a solid phase extraction and high-performance liquid chromatography. Fish Sci. 2001;67(6):1163-8.

41. Weissbach $H$, Waalkes TP, Udenfriend $\mathrm{S}$. A simplified method for measuring serotonin in tissues; simultaneous assay of both serotonin and histamine. J biol Chem. 1958;230(2):865-71.

42. Werner J, Grześkowiak T, Zgoła-Grześkowiak A, Stanisz E. Recent trends in microextraction techniques used in determination of arsenic species. TrAC Trends Anal Chem. 2018;105:121-36. https://doi.org/10.1016/j.trac.2018.05.006.

43. Alsharif AM, Tan G, Choo Y, Lawal A. Liquid phase microextraction for analysis of mycotoxins in food samples: review. Res J Chem Environ Sci. 2015;3(6):5-21.

44. Arthur $\mathrm{CL}$, Pawliszyn J. Solid phase microextraction with thermal desorption using fused silica optical fibers. Anal Chem. 1990;62(19):2145-8.

45. Pawliszyn J. Sampling and sample preparation for field and laboratory: fundamentals and new directions in sample preparation. Amsterdam: Elsevier; 2002.

46. Górecki T, Yu X, Pawliszyn J. Theory of analyte extraction by selected porous polymer SPME fibres. Analyst. 1999;124(5):643-9.

47. Popp P, Bauer C, Wennrich L. Application of stir bar sorptive extraction in combination with column liquid chromatography for the determination of polycyclic aromatic hydrocarbons in water samples. Anal Chim Acta. 2001;436(1):1-9.

48. Kolahgar B, Hoffmann A, Heiden AC. Application of stir bar sorptive extraction to the determination of polycyclic aromatic hydrocarbons in aqueous samples. J Chromatogr A. 2002;963(1-2):225-30.

49. Abdel-Rehim M. New trend in sample preparation: on-line microextraction in packed syringe for liquid and gas chromatography applications: I. Determination of local anaesthetics in human plasma samples using gas chromatography-mass spectrometry. J Chromatogr B. 2004;801(2):317-21. https://doi.org/10.1016/j.jchromb.2003.11.042.

50. El-Beqqali A, Kussak A, Abdel-Rehim M. Fast and sensitive environmental analysis utilizing microextraction in packed syringe online with gas chromatography-mass spectrometry: determination of polycyclic aromatic hydrocarbons in water. J Chromatogr A. 2006;1114(2):2348. https://doi.org/10.1016/j.chroma.2006.02.024.

51. Huang J, Gan N, Lv F, Cao Y, Ou C, Tang H. Environmentally friendly solid-phase microextraction coupled with gas chromatography and mass spectrometry for the determination of biogenic amines in fish samples. J Sep Sci. 2016;39(22):4384-90. https://doi.org/10.1002/ jssc.201600893.

52. Aflaki F, Ghoulipour V, Saemian N, Sheibani S. Biogenic amine contents in non-alcoholic beers: screening and optimization of derivatization. Food Anal Methods. 2014;7(3):713-20.

53. Alizadeh N, Kamalabadi M, Mohammadi A. Determination of histamine and tyramine in canned fish samples by headspace solid-phase microextraction based on a nanostructured polypyrrole fiber followed by lon mobility spectrometry. Food Anal Methods. 2017;10(9):30018. https://doi.org/10.1007/s12161-017-0860-z.

54. Naing NN, Tan SC, Lee HK. 16-micro-solid-phase extraction. In: Poole CF, editor. Solid-phase extraction. Elsevier; 2020. p. $443-71$.

55. Seidi S, Tajik M, Baharfar M, Rezazadeh M. Micro solid-phase extraction (pipette tip and spin column) and thin film solid-phase microextraction: miniaturized concepts for chromatographic analysis. TrAC Trends Anal Chem. 2019;118:810-27. https://doi.org/10.1016/j.trac. 2019.06.036.

56. Basheer C, Wong W, Makahleh A, Tameem AA, Salhin A, Saad B, et al. Hydrazone-based ligands for micro-solid phase extraction-high performance liquid chromatographic determination of biogenic amines in orange juice. J Chromatogr A. 2011;1218(28):4332-9. https:// doi.org/10.1016/j.chroma.2011.04.073.

57. Baumbach J. Process analysis using ion mobility spectrometry. Anal Bioanal Chem. 2006;384(5):1059-70.

58. Sarafraz-Yazdi A, Amiri A. Liquid-phase microextraction. TrAC Trends Anal Chem. 2010;29(1):1-14. https://doi.org/10.1016/j.trac.2009.10. 003.

59. Rutkowska M, Płotka-Wasylka J, Sajid M, Andruch V. Liquid-phase microextraction: a review of reviews. Microchem J. $2019 ; 149: 103989$.

60. Yamini Y, Rezazadeh M, Seidi S. Liquid-phase microextraction-the different principles and configurations. TrAC Trends Anal Chem. 2019;112:264-72. https://doi.org/10.1016/j.trac.2018.06.010.

61. Liu H, Dasgupta PK. Analytical chemistry in a drop. Solvent extraction in a microdrop. Anal Chem. 1996;68(11):1817-21. https://doi.org/ 10.1021/ac960145h.

62. Jeannot MA, Cantwell FF. Solvent microextraction into a single drop. Anal Chem. 1996;68(13):2236-40. https://doi.org/10.1021/ac960 $042 z$.

63. He Y, Lee HK. Liquid-phase microextraction in a single drop of organic solvent by using a conventional microsyringe. Anal Chem. 1997;69(22):4634-40. https://doi.org/10.1021/ac970242q.

64. Ramos L. Critical overview of selected contemporary sample preparation techniques. J Chromatogr A. 2012;1221:84-98.

65. Rutkowska M, Płotka-Wasylka J, Sajid M, Andruch V. Liquid-phase microextraction: a review of reviews. Microchem J. $2019 ; 149: 103989$. https://doi.org/10.1016/j.microc.2019.103989.

66. Ocaña-González JA, Fernandez-Torres R, Bello-López MÁ, Ramos-Payan M. New developments in microextraction techniques in bioanalysis. A review. Anal Chimica Acta. 2016;905:8-23.

67. Almeida C, Fernandes J, Cunha S. A novel dispersive liquid-liquid microextraction (DLLME) gas chromatography-mass spectrometry (GC-MS) method for the determination of eighteen biogenic amines in beer. Food Control. 2012;25(1):380-8.

68. Donthuan J, Yunchalard S, Srijaranai S. Ultrasound-assisted dispersive liquid-liquid microextraction combined with high performance liquid chromatography for sensitive determination of five biogenic amines in fermented fish samples. Anal Methods. 2014;6(4):1128-34. https://doi.org/10.1039/C3AY41572D.

69. Wu H, Li G, Liu S, Ji Z, Zhang Q, Hu N, et al. Simultaneous determination of seven biogenic amines in foodstuff samples using one-step fluorescence labeling and dispersive liquid-liquid microextraction followed by HPLC-FLD and method optimization using response surface methodology. Food Anal Methods. 2015;8(3):685-95. https://doi.org/10.1007/s12161-014-9943-2.

70. Mohammadi M, Kamankesh M, Hadian Z, Mortazavian AM, Mohammadi A. Determination of biogenic amines in cheese using simultaneous derivatization and microextraction method followed by gas chromatography-mass spectrometry. Chromatographia. 2017;80(1):11926. https://doi.org/10.1007/s10337-016-3217-7. 
71. Kamankesh M, Mohammadi A, Mollahosseini A, Seidi S. Application of a novel electromembrane extraction and microextraction method followed by gas chromatography-mass spectrometry to determine biogenic amines in canned fish. Anal Methods. 2019;11(14):1898-907. https://doi.org/10.1039/c9ay00224c.

72. Pedersen-Bjergaard S, Rasmussen KE. Electrokinetic migration across artificial liquid membranes: new concept for rapid sample preparation of biological fluids. J Chromatogr A. 2006;1109(2):183-90.

73. Seidi S, Yamini Y, Heydari A, Moradi M, Esrafili A, Rezazadeh M. Determination of thebaine in water samples, biological fluids, poppy capsule, and narcotic drugs, using electromembrane extraction followed by high-performance liquid chromatography analysis. Anal Chim Acta. 2011;701(2):181-8.

74. Raynie DE. Modern extraction techniques. Anal Chem. 2010;82(12):4911-6.

75. Huddleston JG, Visser AE, Reichert WM, Willauer HD, Broker GA, Rogers RD. Characterization and comparison of hydrophilic and hydrophobic room temperature ionic liquids incorporating the imidazolium cation. Green Chem. 2001;3(4):156-64.

76. Ma J, Hong X. Application of ionic liquids in organic pollutants control. J Environ Manage. 2012;99:104-9.

77. Elik A, Altunay N, Gürkan R. Ultrasound-assisted low-density solvent-based dispersive liquid-liquid microextraction coupled to spectrophotometry for the determination of low levels of histamine in fish and meat products. Food Anal Methods. 2019;12(2):489-502. https:// doi.org/10.1007/s12161-018-1380-1.

78. Nancarrow $\mathrm{P}$, Mohammed $\mathrm{H}$. Ionic liquids in space technology_current and future trends. ChemBioEng Reviews. 2017;4(2):106-19. https://doi.org/10.1002/cben.201600021.

79. Cao D, Xu X, Xue S, Feng X, Zhang L. An in situ derivatization combined with magnetic ionic liquid-based fast dispersive liquid-liquid microextraction for determination of biogenic amines in food samples. Talanta. 2019;199:212-9. https://doi.org/10.1016/j.talanta.2019. 02.065 .

80. Jinadasa BKKK, Moreda-Piñeiro A, Fowler SW. Chapter 36-application of combined extraction and microextraction techniques for food waste. In: Bhat R, editor. Valorization of agri-food wastes and by-products. Academic Press; 2021. p. 711-22.

81. Werner J, Grześkowiak T, Zgoła-Grześkowiak A, Stanisz E. Recent trends in microextraction techniques used in determination of arsenic species. Trends Anal Chem. 2018;105:121.

82. Jinadasa BKKK, Monteau F, Morais S. Critical review of micro-extraction techniques used in the determination of polycyclic aromatic hydrocarbons in biological, environmental and food samples. Food Addit Contam Part A. 2020. https://doi.org/10.1080/19440049.2020. 1733103.

83. Papageorgiou M, Lambropoulou D, Morrison C, Namieśnik J, Płotka-Wasylka J. Direct solid phase microextraction combined with gas chromatography-mass spectrometry for the determination of biogenic amines in wine. Talanta. 2018;183:276-82. https://doi.org/10. 1016/j.talanta.2018.02.006.

84. Tameem AA, Saad B, Makahleh A, Salhin A, Saleh MI. A 4-hydroxy-N'-[(E)-(2-hydroxyphenyl)methylidene]benzohydrazide-based sorbent material for the extraction-HPLC determination of biogenic amines in food samples. Talanta. 2010;82(4):1385-91. https://doi.org/10. 1016/j.talanta.2010.07.004.

85. Molaei R, Tajik H, Moradi M, Forough M. Application of novel Fe $\mathrm{O}_{4}$-g-GO-g-RAFT agent nanoabsorbents for D-SPME of biogenic amines in smoked fish. J Food Compos Anal. 2020;87: 103400. https://doi.org/10.1016/j.jfca.2019.103400.

86. Wojnowski W, Namieśnik J, Płotka-Wasylka J. Dispersive liquid-liquid microextraction combined with gas chromatography-mass spectrometry for in situ determination of biogenic amines in meat: estimation of meat's freshness. Microchem J. 2019;145:130-8. https://doi. org/10.1016/j.microc.2018.10.034.

87. Jia S, Ryu Y, Kwon SW, Lee J. An in situ benzoylation-dispersive liquid-liquid microextraction method based on solidification of floating organic droplets for determination of biogenic amines by liquid chromatography-ultraviolet analysis. J Chromatogr A. 2013;1282:1-10.

88. Saaid M, Saad B, Ali ASM, Saleh MI, Basheer C, Lee HK. In situ derivatization hollow fibre liquid-phase microextraction for the determination of biogenic amines in food samples. J Chromatogr A. 2009;1216(27):5165-70.

89. Aflaki F, Ghoulipour V, Saemian N, Salahinejad M. A simple method for benzoyl chloride derivatization of biogenic amines for high performance liquid chromatography. Anal Methods. 2014;6(5):1482-7.

90. Donthuan J, Yunchalard S, Srijaranai S. Vortex-assisted surfactant-enhanced-emulsification liquid-liquid microextraction of biogenic amines in fermented foods before their simultaneous analysis by high-performance liquid chromatography. J Sep Sci. 2014;37(21):316473. https://doi.org/10.1002/jssc.201400570.

91. Płotka-Wasylka J, Simeonov V, Namieśnik J. An in situ derivatization-dispersive liquid-liquid microextraction combined with gaschromatography-mass spectrometry for determining biogenic amines in home-made fermented alcoholic drinks. J Chromatogr A. 2016;1453:10-8. https://doi.org/10.1016/j.chroma.2016.05.052.

Publisher's Note Springer Nature remains neutral with regard to jurisdictional claims in published maps and institutional affiliations. 